\title{
Transportables Kransystem für Probenentnahmearbeiten in beliebigen Booten
}

\author{
Von H. Ambühl und H. Bührer \\ EAWAG Dübendorf \\ Manuskript eingegangen am 11. November 1985
}

ABSTRACT

Transportable crane system for use in sampling from any boat A relatively light, demountable and transportable crane system is described. The crane is head height, rotatable and can be fastened to any suitable boat. By attaching different booms, all types of limnological test equipment can be suspended. A newly developed cable feeding mechanism is integrated into the cable spool mounting. Motorisation enables loads of up to $30 \mathrm{~kg}$ to be raised and permits the crane to operate as a winch.

Die Methodik der Entnahme von Wasser-, Sediment- und Organismenproben aus Seen befindet sich heute auf einem modernen Stand; eine breite Auswahl von altbewährten sowie neu entwickelten Entnahmegeräten steht zur Verfügung. Um diese zu bewegen, sind Kraneinrichtungen und Seilwinden erforderlich. Derartige Geräte finden sich jedoch nur auf den speziell eingerichteten Arbeitsbooten der limnologischen Institute und einzelner Gewässerschutzfachstellen; Schulen usw. sind dagegen eher bescheiden ausgerüstet, meist nur mit einfachen Handwinden, welche den oft schweren modernen Arbeitsgeräten nicht gewachsen sind.

Zur Probenentnahme vom Boot aus arbeitet man am besten stehend; dies ist nur möglich mit einem stehhohen und schwenkbaren Kran. Wir haben ein System von transportablen Kränen entwickelt, das, mit den Vorzügen der fest eingebauten Kräne versehen (und auch als solche verwendbar), in jedem beliebigen Boot eingesetzt werden kann, und dies in Hand- als auch in motorisiertem Betrieb.

Es handelt sich um eine Weiterentwicklung unserer früher beschriebenen Durchlaufwinde [1]. Der Kran ruht auf einem Gelenk mit horizontaler Achse, welches erlaubt, die Kranbasis der jeweiligen Schräge der Bordwand anzupassen (Abb.8). Die Kransäule ist drehbar gelagert; der Kran kann um seine vertikale Achse gedreht werden. Der Ausleger lässt sich auf verschiedene Neigungen und Längen einstellen (z. B. hoch und nahe beim Boot für schwere Geräte, weit ausladend für leichte Geräte wie Unterwasserphotometer usw., Abb. 1 und 2). Für besonders lange Arbeitsgeräte (Sedimentlote, Grazingkammern) wurde ein speziell hohes Auslegergestänge entworfen. Obschon aus Gewichtsgründen 

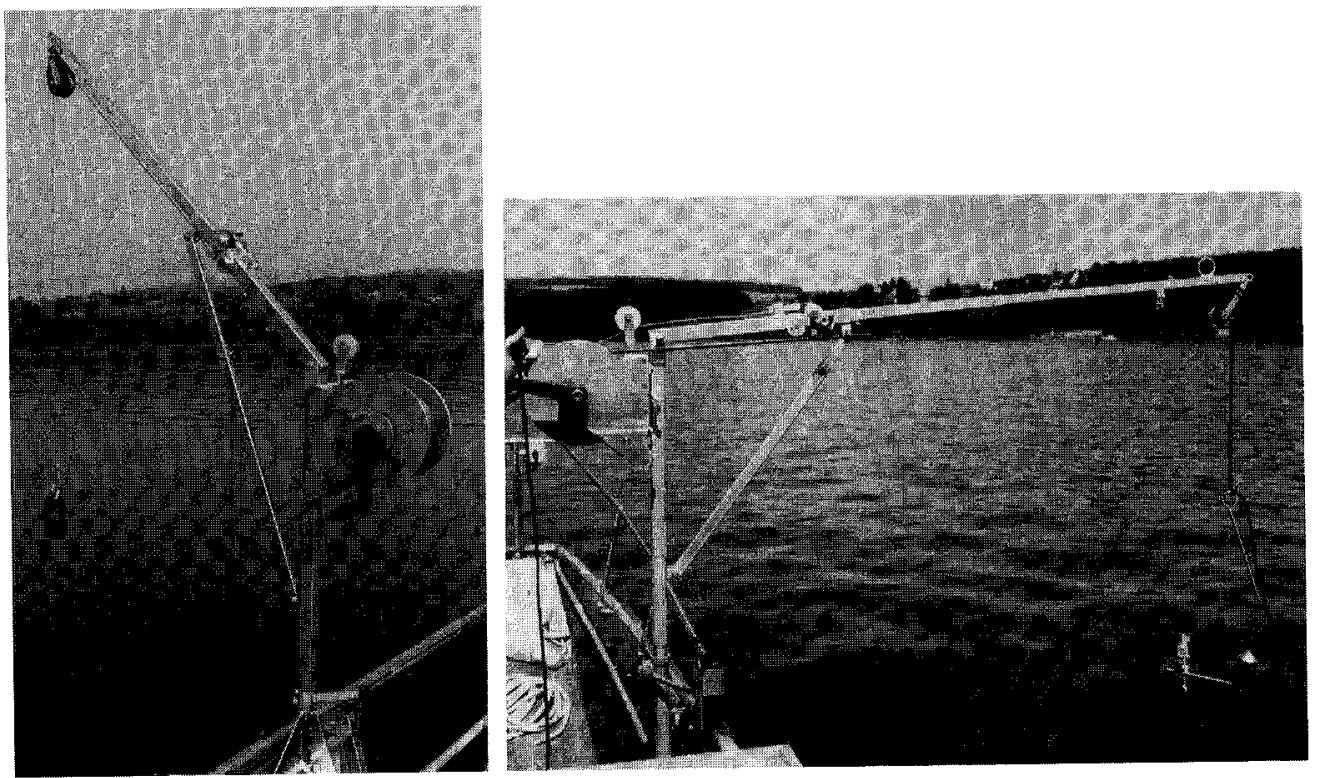

Abb. 1. Handbetriebener Probenkran in der am meisten verwendeten Stellung, mit normalem Ausleger. Figure 1. Hand operated prototype crane in its most usual position with the normal boom.

Abb. 2. Handkran mit verlängertem Ausleger (unter Verwendung des Auslegereinsatzes von Abb. 3) für Strahlungsmeßsonden.

Figure 2. Hand crane with extension boom (used with the boom assembly in fig. 3), e.g. for radiation sensors.

sehr leicht gebaut, können damit doch grosse Kräfte abgefangen werden (z. B. während der Extraktion von Bohrkernen), wenn der Ausleger mit einer Leine auf die gegenüberliegende Bordwand abgespannt wird (Abb. 3). Um das Gesamtgewicht möglichst niedrig zu halten, wurde zum vorneherein auf Stabilitätsreserve verzichtet.

Die Seiltrommel wird mit Handkurbeln bewegt und mit Klinken arretiert. Im motorisierten Modell wird sie mit einem Elektromotor von 200 Watt Leistung angetrieben (Abb. 4). Eine im Getriebemotor eingebaute Magnetbremse hat sich im praktischen Einsatz dank der Selbsthaltung des verwendeten Schneckengetriebes als überflüssig erwiesen. Der Keilriemen zwischen der Getriebewelle und der Trommelachse dient mit Hilfe einer beweglichen Andruckrolle auch als Kupplung. Die eine Handkurbel kann leicht durch einen Capstan (Spill) ausgetauscht werden. Wird das Kranseil aus dem Ausleger zurückgezogen und auf der Trommel festgemacht, so kann die Winde bei laufendem Motor als kräftige «Winsch» (Durchlaufwinde) für Seile, Leinen usw. benützt werden (Abb. 5).

Der Ausleger trägt eine Messrolle aus rostfreiem Hartmetall, welche den abgespulten Umfang in Metern und Dezimetern auf einem mechanischen Zählwerk anzeigt (für den rauhen, nassen Feldbetrieb sind die leicht ersetzbaren mechanischen Anzeigen noch immer am geeignetsten; Abb.6). Das Kranseil (rostfreies Stahlseil von 2 bzw. 2,5 mm Durchmesser und mit Längen bis $300 \mathrm{~m}$ ) wird durch eine Fadenführung auf der Trommel 

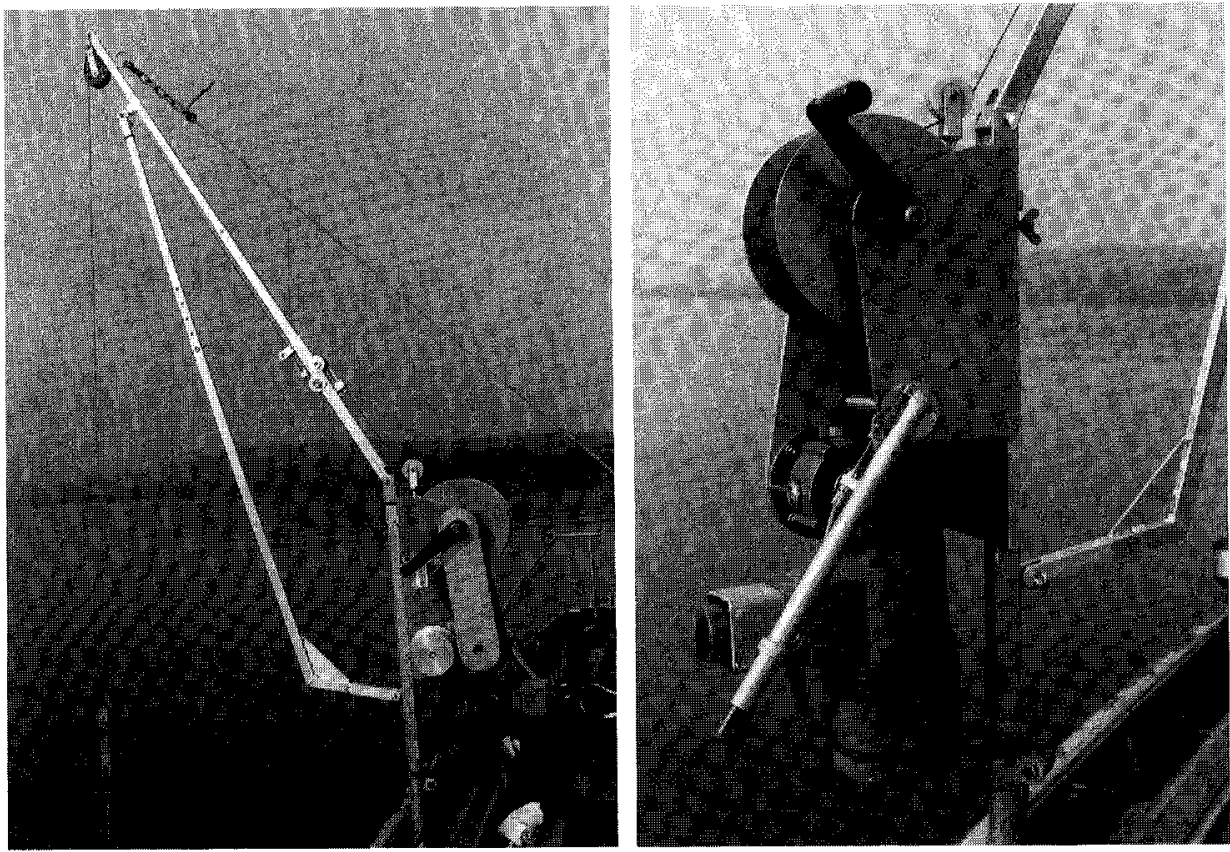

Abb. 3. Hoher Ausleger für lange Geräte. Die hinter der Seilrolle befestigte Leine führt zur anderen Bordwand. Sie fängt grosse Beanspruchungen ab.

Figure 3. Higher boom for longer instruments. The line fastened behind the cable pulley leads to the other side of the ship, conferring much greater stability.

Abb. 4. Motorgetriebener Probenkran. Sämtliche Auslegerkombinationen (Abb. 1-3) sind hier in gleicher Weise verwendbar.

Figure 4. Motor operated prototype crane. The complete range of boom constructions (fig. 1-3) can be used here in the same way.

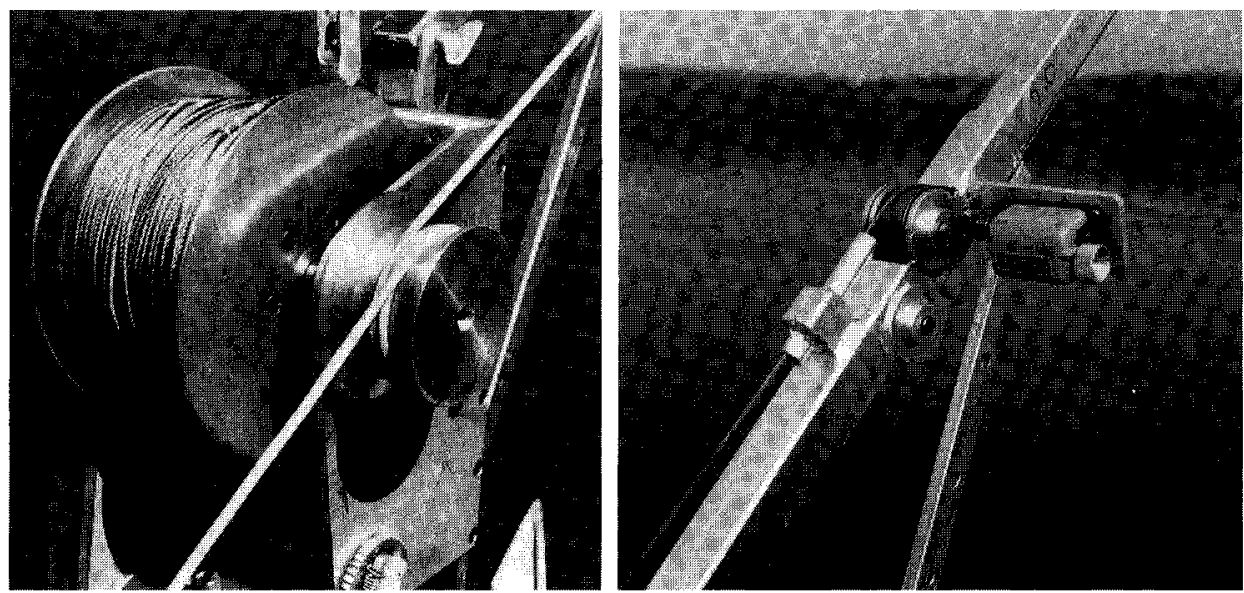

Abb. 5. Motorkran; die rechte Handkurbel ist durch ein Spill ersetzt. Das Drahtseil ist auf der Trommel festgemacht.

Figure 5. Motorized crane. The right hand handle has been replaced by a capstan. The wire cable is fastened to the cable spool.

Abb. 6. Meterzählwerk: Die obere Umlenkrolle ist zugleich Messrolle. Das Zählwerk ist handelsüblich und leicht ersetzbar.

Figure 6. Meter counter. The counter is commercially available and easily replaced. 


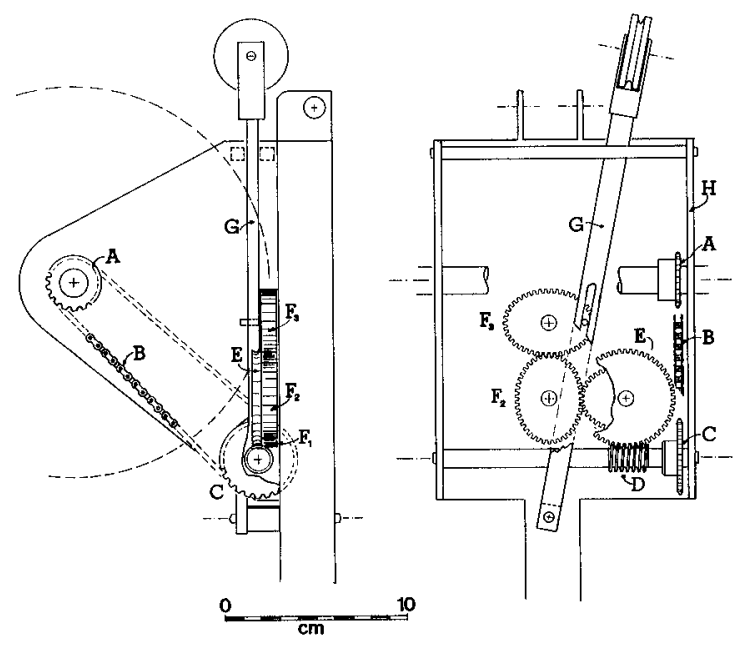

Abb. 7. Prinzipskizze der Seilführung (Ansicht und Seitensicht): Ein Zahnrad A auf der Achse der Kabeltrommel treibt über eine Rollenkette B die Achse C an. Die Schnecke D treibt das Schneckenzahnrad E und damit das erste Ovalzahnrad $F_{1}$. Dieses treibt die Ovalräder $F_{2}$ und $F_{3} . F_{1}$ bewegt sich linear mit der

Trommeldrehung, $F_{2}$ bewegt sich in der hier dargestellten Lage am raschesten, nach $90^{\circ}$ am langsamsten. Dieser Effekt ist durch die nochmalige «Transformation» auf $F_{3}$ so stark, dass sich der Hebel $G$ nahezu linear zur Drehung der Seiltrommel hin- und herbewegt. Die Seiltrommel ist in der Skizze weggelassen, ebenso Teile des Verteilhebels und des Schneckenzahnrades. In der Seitensicht ist die Seitenwange H weggelassen.

Figure 7. Technical diagram of cable mechanism (front and side view): The cog-wheel (A) on the axle of the cable spool drives via chain (B) the axle (C). The worm-gear (D) drives the worm-cog-wheel (E) and the first oval $\operatorname{cog} F_{1}$. This drives the oval cogs $F_{2}$ and $F_{3} . F_{1}$ moves linearly to the spool motion while $F_{2}$ moves fast in the depicted position and slowly after $90^{\circ}$ rotation. This effect is amplified by the supplementary 'transformation' of $F_{3}$, such that the crank $(G)$ moves from side to side nearly linearly to the rotation of the cable spool. The latter has been omitted from the diagram as have parts of the distribution crank and of the worm-cog-wheel. In the side view, the wall $H$ has also been omitted.

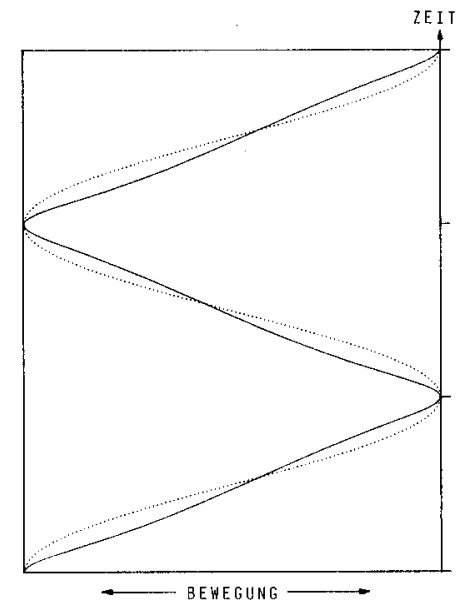

Abb. 7a. Ausgezogene Kurve: Bewegung der Seilführung, gesteuert durch drei Ovalzahnräder 1,35:1. Punktierte Linie: reine Sinusbewegung.

Figure 7a. Solid curve: motion of the cable crank driven by three oval cogs 1,35:1. Dotted curve: pure sine motion. 
gleichmässig verteilt. Anstelle der bisher gebräuchlichen Seilführung mit Steuerkurve haben wir einen Mechanismus entwickelt, welcher eine genügend lineare Links-rechtsBewegung mit Hilfe von drei ovalen Zahnrädern realisiert (Abb. 7).

Diese Zahnräder, übrigens die einzigen nicht handelsüblichen Bauteile des ganzen Systems, sind folgendermassen dimensioniert: Das Verhältnis der Achsen des Berührungskörpers beträgt 1,35:1. Anzahl der Zähne: 42, nach Modul 1 bemessen. Die Berührungskurve («Teilkurve», abgeleitet vom «Teilkreis» der kreisförmigen Zahnräder) wird durch die Funktion

$$
\frac{x^{n}}{a^{n}}+\frac{y^{n}}{b^{n}}=1, \quad \text { wobei } a=23,88 \quad b=17,6089 \quad n=2,06767,
$$

beschrieben. Die Berührungskurve ist somit keine Ellipse. In rechtwinkligen Koordinaten, welche den Nachbau ohne weiteres erlauben, kann sie für ein Viertelrad folgendermassen beschrieben werden:

\begin{tabular}{llll}
\hline $\begin{array}{l}\text { Aussen } \\
\text { Xa }\end{array}$ & Ya & Innen & Yi \\
\hline 1,61 & 18,66 & $\mathrm{Xi}$ & 16,49 \\
4,83 & 18,38 & 1,52 & 16,23 \\
8,02 & 17,80 & 4,55 & 15,69 \\
11,14 & 16,88 & 7,52 & 14,84 \\
14,14 & 15,61 & 10,41 & 13,67 \\
16,98 & 13,95 & 13,18 & 12,16 \\
19,56 & 11,88 & 15,75 & 10,31 \\
21,77 & 9,40 & 18,07 & 8,11 \\
23,47 & 6,52 & 20,02 & 5,61 \\
24,54 & 3,33 & 21,51 & 2,88 \\
24,88 & 0,00 & 22,42 & 0,00 \\
\hline
\end{tabular}

Angaben in $\mathrm{mm}$.

Die durch je ein Datenpaar definierten Punkte bezeichnen die Mitte der äusseren bzw. inneren Fläche des jeweiligen Zahnes. Die korrespondierenden Paare (aussen-innen) geben auch die Richtung der Zähne an. Diese sind senkrecht zur Tangente angeordnet. Sie werden mit einem Modulfräser $15^{\circ}$ Modul 1 in den Körper (definiert durch die Punkte «aussen») eingefräst. Der Umfang auf der Berührungskurve beträgt $42 \times \pi \mathrm{mm}$.

Die Hin- und Herbewegung, die aus dieser Anordnung resultiert, ist nicht streng linear, aber für den praktischen Bedarf weitaus genügend (siehe Abb. 7a). So wird ein Drahtseil von $300 \mathrm{~m}$ Länge gleichmässig auf der Trommel verteilt.

Dieses System benötigt sehr wenig Raum und arbeitet, da es keine heiklen Bauelemente enthält, störungsfrei.

Wie eingangs erwähnt, ist die ganze Einrichtung auf einem Basisbrett montiert, welches mit zwei Schraubzwingen am Bordrand festgeklemmt wird (Abb.8). Ein dritter, für die Stabilität wichtiger Fixpunkt befindet sich in der Mitte am unteren Rand des ausziehbaren, jeder Bordhöhe anzupassenden Brettes. Mit einer Spreize aus flachem Stahlrohr wird das Brett an die Bordwand angepresst; die Spreize selber wird an der gegenüberliegenden Bordwand in gleicher Weise verankert (Abb.9). Die Arbeitsboote unseres Instituts (Standorte Vierwaldstättersee, Greifensee, Hallwilersee) wurden mit festen Kranpodesten versehen, in welche die Kräne nach Bedarf eingesetzt werden (Abb. 10).

Für die Konstruktion wurden (mit Ausnahme der ovalen Zahnräder) ausschliesslich handelsübliche Konstruktionsmaterialien verwendet. Der Getriebemotor stammt aus dem normalen Sortiment einer Spezialfirma ${ }^{1}$ ). Als Stromquelle dient ein E-Generator.

1) Detaillierte Informationen sind bei den Autoren erhältlich. 

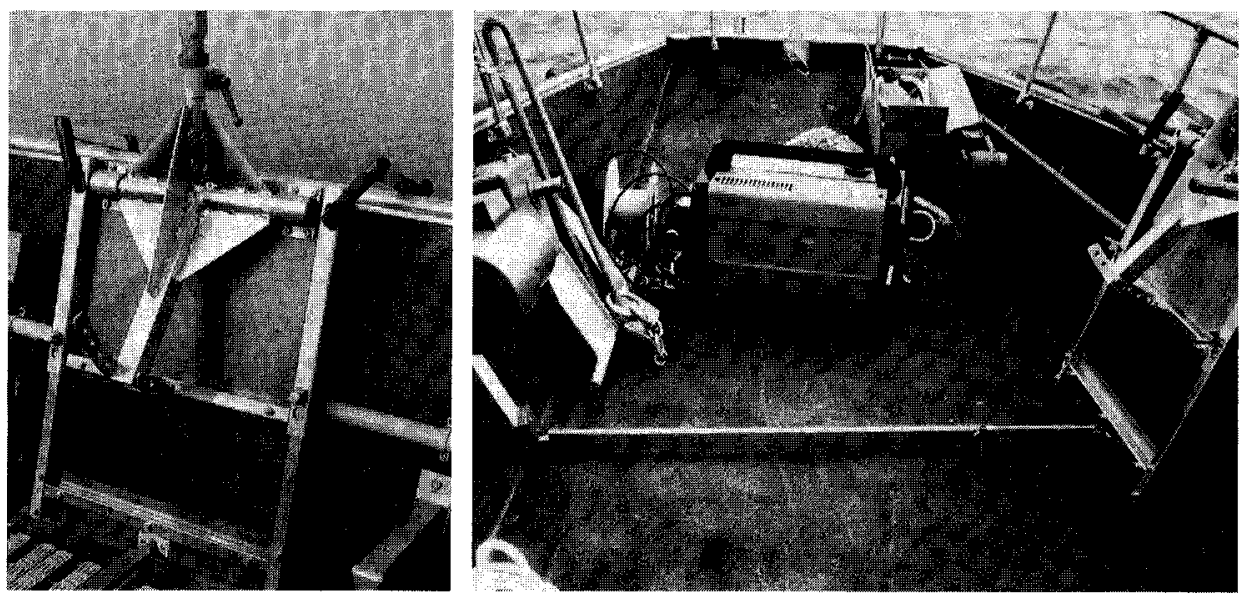

Abb. 8. Kranpodest. Der ausziehbare untere Teil kann jeder Bordhöhe angepasst werden. Die Neigung ist einstellbar, um die Kransäule in vertikaler Lage zu fixieren. Unten ist die Spreize sichtbar, oben die beiden Schraubzwingen.

Figure 8. Crane podium. The extendable lower portion makes it suitable for any board height. The angle is adjustable so that the crane column can be fixed in the vertical position. At the bottom, the prop can be seen, and above, both screw clamps.

Abb. 9. Situation: Die Kranbasis (rechts) wird unten gegen die Bordwand gepresst. Die Spreize wird an beiden Enden in Bohrungen gehalten.

Figure 9. The crane base (right) is fastened underneath the ships side. The prop is held at both ends in guide holes.

Der 220-V-Generatorbetrieb hat gegenüber dem Niedervolt-(bzw. Batterie-)betrieb den Vorteil, dass Motoren mit hohem Wirkungsgrad verwendet werden können (12-V-Motoren sind bei gleicher Leistungsabgabe rund doppelt so schwer) und dass der stets problematische Transport von Batterien und deren Wartung entfällt. Auf Direktantrieb mit einem Verbrennungsmotor wurde aus Gründen der Betriebssicherheit und vor allem der Arbeitshygiene verzichtet.

Die Kräne sind aus dünnwandigem, leichtem Profilstahlrohr in handelsüblichen Grössen gefertigt. Stahl ist billig und lässt sich problemlos bearbeiten und verbinden, ferner durch galvanische Behandlung leicht und dauerhaft vor Rost schützen. Aus Gewichtsgründen wird der Motor separat transportiert und erst auf dem Boot am aufgebauten Kran montiert. Beide Kräne sind für eine Last von $30 \mathrm{~kg}$ ausgelegt; diese Last wird von der Motorwinde ohne Schwierigkeiten gehoben. Bei grösserer Last kann mit den Handkurbeln nachgeholfen werden. Die Drehzahl des Kollektormotors ist zwar lastabhängig, dafür ist er überlastungssicher; er kann kurzzeitig abgewürgt werden, ohne Schaden zu nehmen. Die Hubgeschwindigkeit beträgt bei normaler Last und voller Seiltrommel 0,75-1 m/s. Die einzelnen Bauelemente haben folgende Gewichte: Handkran mit $300 \mathrm{~m}$ Stahlseil 2,0 mm (ohne Podest): 19,5 kg; Motorkran (150 m Stahlseil 2,5 mm, ohne Podest, ohne Motor: 16,25 kg, Getriebemotor: 10,75 kg; Kranpodest: 9,5 kg).

Für besondere Anwendungen, z. B. gleichmässiges Bewegen von Meßsonden, steht ein Asynchron-Getriebemotor mit lastunabhängiger, konstanter Drehzahl zur Verfügung. 


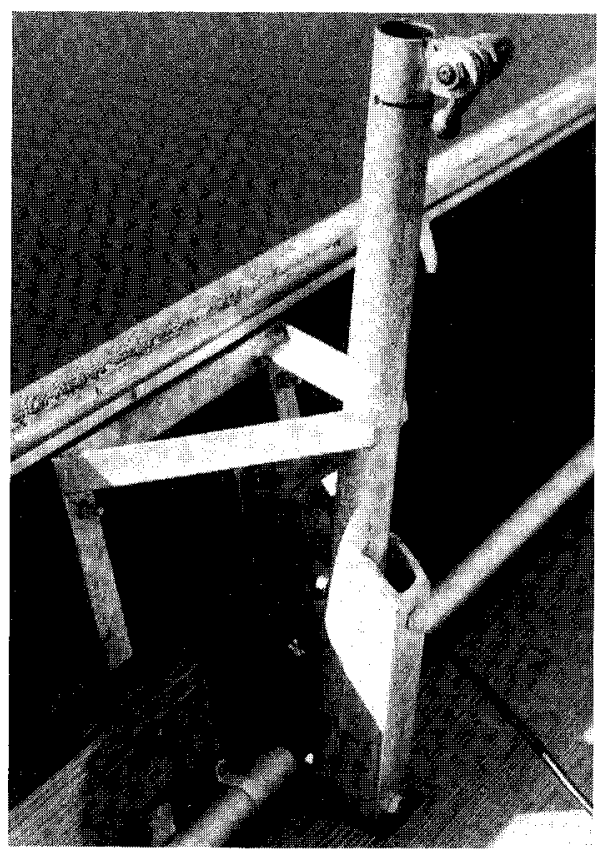

Abb. 10. Beispiel eines fest eingebauten Kranpodestes. Diese Podeste sind der Bauart der Boote individuell angepasst.

Figure 10. Example of a permanently installed crane podium. The podiums are individually suited to each boat type.

\section{Zusammenfassung}

Es wird ein System von relativ leichten, demontablen und transportablen Kränen beschrieben. Diese sind stehhoch, drehbar und können in jedem beliebigen Boot befestigt werden. Dank verschiedenen Auslegern können alle Typen von limnologischen Arbeitsgeräten bewegt werden. Ein neu entwickelter Seilführungs-Mechanismus ist platzsparend in die Trommellagerung integriert. Eine motorisierte Ausführung hebt Lasten bis $30 \mathrm{~kg}$ und kann auch als Durchlaufwinde (Winsch) verwendet werden.

\section{VERDANKUNG}

Die mechanischen Arbeiten wurden von der Werkstatt der Technischen Versuchsstation der EAWAG ausgefürt. Die Autoren danken Eugen Schwager und W. Häfliger für ihre zuverlässige Arbeit und besonders auch Dipl. Ing. H. Burkhalter für die Vorbereitung und H. Schälchli für die Herstellung der ovalen Zahnräder, ferner P. Schlup für die wiederholten photographischen Arbeiten und Anthony Mason für die englische Übersetzung.

\section{LITERATURVERZEICHNIS}

1 Ambühl, H.: Eine transportable Motor-Durchlaufwinde (Winch) zum Einsatz in beliebigen Booten. Schweiz. Z. Hydrol. 45, 495-497 (1983).

Adresse der Autoren: EAWAG, Fachabteilung Hydrobiologie/Limnologie, Überlandstrasse 133, CH-8600 Dübendorf (Switzerland). 